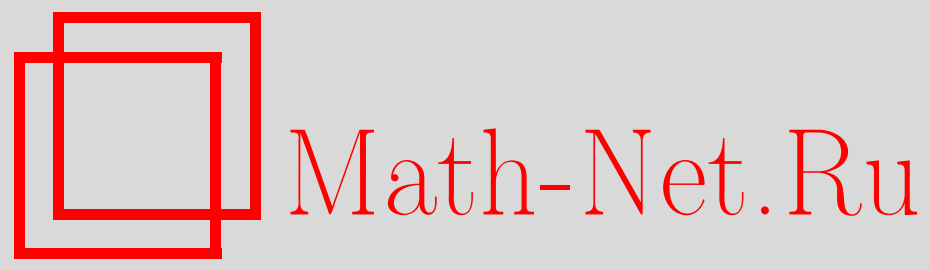

И. А. Тайманов, Представление Вейерштрасса за-

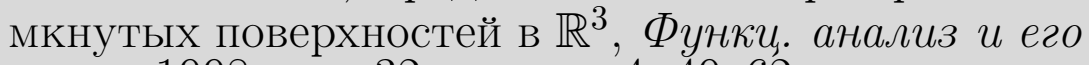
прил., 1998, том 32, выпуск 4, 49-62

DOI: https://doi.org/10.4213/faa437

Использование Общероссийского математического портала MathNet.Ru подразумевает, что вы прочитали и согласны с пользовательским соглашением

http://www . mathnet.ru/rus/agreement

Параметры загрузки:

IP : 3.91 .87 .62

26 апреля 2023 г., 16:28:25

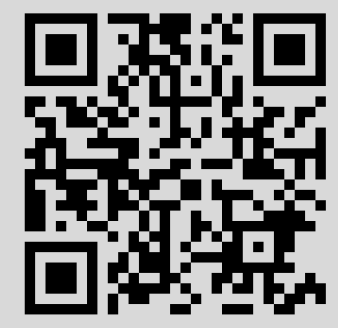


Функииональный анализ и его приложения

1998, т. 32, вып. 4, с. 49-62

УДК $514.752 .43+517.984$

\section{Представление Вейерштрасса замкнутых поверхностей в $\mathbb{R}^{3} \star$}

(C) 1998. И. А. ТАЙМАНОВ

\section{§1. Введение}

Данная статья является продолжением работ $[16,17]$. Изложенные здесь результаты распространяют на произвольные поверхности результаты, полученные в [17] для поверхностей вращения, и на протяжении последнего года они излагались в многочисленных докладах автора, пока не были анонсированы в [18]. Это

- построение глобального представления Вейерштрасса для произвольной замкнутой ориентированной поверхности рода $g \geqslant 1$, погруженной в $\mathbb{R}^{3}(\S 2)$;

- построение спектра Вейерштрасса для тора, погруженного в $\mathbb{R}^{3}$, и обсуждение его геометрических свойств (§3);

- построение конечнозонных поверхностей (§4).

В $\S 5$ мы обсуждаем связь этих конструкций с гипотезой Уиллмора.

Указанное представление устанавливает взаимно однозначное соответствие между

a) решениями уравнений $\mathscr{D} \psi=0$ для операторов Дирака $\mathscr{D}$ на спинорных расслоениях специального вида над замкнутыми ориентированными поверхностями $\Sigma$ постоянной кривизны

и

б) погружениями универсальных накрытий поверхностей $\Sigma$ в $\mathbb{R}^{3}$, такими, что их отображения Гаусса пропускаются через $\Sigma$.

Это дает возможность описать пространство модулей всех погружений поверхностей в $\mathbb{R}^{3}$ в терминах операторов Дирака в спинорных расслоениях над поверхностями постоянной кривизны и указать новые геометрические инварианты поверхностей в терминах спектра оператора Дирака.

\section{§2. Представление Вейерштрасса}

\section{1. Локальное представление Вейерштрасса.}

Лемма 1. Пусть $W-$ односвлзнал область в $\mathbb{C} u \psi=\left(\psi_{1}, \psi_{2}\right): W \rightarrow \mathbb{C}^{2}$ удовлетворяет уравнению

$$
\mathscr{D} \psi=0,
$$

^ Работа поддержана РФФИ (грант 96-15-96877) и SFB 288. 


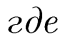

$$
\mathscr{D}=\left(\begin{array}{cc}
0 & \partial \\
-\bar{\partial} & 0
\end{array}\right)+\left(\begin{array}{cc}
U & 0 \\
0 & U
\end{array}\right)
$$

и функция $U(z, \bar{z})$ вещественна. Тогда формуль

$$
\begin{aligned}
& X^{1}(z, \bar{z})=\frac{i}{2} \int_{\gamma}\left(\left(\bar{\psi}_{2}^{2}+\psi_{1}^{2}\right) d z^{\prime}-\left(\bar{\psi}_{1}^{2}+\psi_{2}^{2}\right) d \bar{z}^{\prime}\right), \\
& X^{2}(z, \bar{z})=\frac{1}{2} \int_{\gamma}\left(\left(\bar{\psi}_{2}^{2}-\psi_{1}^{2}\right) d z^{\prime}-\left(\bar{\psi}_{1}^{2}-\psi_{2}^{2}\right) d \bar{z}^{\prime}\right), \\
& X^{3}(z, \bar{z})=\int_{\gamma}\left(\psi_{1} \bar{\psi}_{2} d z^{\prime}+\bar{\psi}_{1} \psi_{2} d \bar{z}^{\prime}\right)
\end{aligned}
$$

задают погружение области $W$ в $\mathbb{R}^{3}$. При этом индуцированная метрика имеет вид $D(z, \bar{z})^{2} d z d \bar{z}$, где $D(z, \bar{z})=\left(\left|\psi_{1}(z, \bar{z})\right|^{2}+\left|\psi_{2}(z, \bar{z})\right|^{2}\right)$, и средняя кривизна равна

$$
H(z, \bar{z})=2 \frac{U(z, \bar{z})}{D(z, \bar{z})} .
$$

Погружение $W \rightarrow \mathbb{R}^{3}$ строится следующим образом. Возьмем точку $z_{0} \in$ $W$ и отобразим ее в $0 \in \mathbb{R}^{3}$. После этого определим $X^{i}(z, \bar{z})$ как значение интеграла (3), взятого вдоль произвольного пути в $W$, соединяющего $z_{0}$ и $z$.

Для $U=0$ это - классическое представление Вейерштрасса минимальных поверхностей.

Видимо, эта лемма принадлежит Эйзенхарту [3], который выписывал (1) как условие только на $\psi_{1}: L \psi_{1}=0$ в терминах оператора

$$
L=\partial \bar{\partial}-\frac{\partial U}{U} \bar{\partial}+U^{2}
$$

В конце 80-х годов Абреш вывел представление Вейерштрасса для поверхностей постоянной средней кривизны, используя оператор (2), и рассматривал глобальное представление для построения точных формул для таких поверхностей рода $g \geqslant 2$. Так как программа построения таких формул до сих пор не реализована, то результаты Абреша были только изложены на конференции в Люмини.

Бобенко показал, что при рассмотрении $\mathbb{R}^{3}$ как пространства мнимых кватернионов некоторые тождества для подвижного репера записываются в виде уравнения Дирака для кватернионнозначных функций, но построение общих поверхностей в терминах собственных функций оператора Дирака и глобализация этой конструкции им не обсуждалась [1].

В [9] Конопельченко рассмотрел «квадратный корень» из (5), т. е. матричный дифференциальный оператор первого порядка (2), для определения деформаций поверхностей, заданных формулами (3), с помощью модифицированных уравнений Веселова-Новикова.

Эта замена двумерного оператора Шрёдингера (5) с потенциалом и магнитным полем на оператор Дирака (2) с потенциалом $U$ делает представление более удобным для приложений. 
Лемма 2. Пусть $W$ - область в $\mathbb{C} u X: W \rightarrow \mathbb{R}^{3}-$ конформное погружение $W$ в $\mathbb{R}^{3}$. Предположим, ито

$$
\frac{\partial X^{3}}{\partial z} \neq 0
$$

в окрестности точки $z_{0} \in W$. Тогда в окрестности точки $z_{0}$ функции

$$
\psi_{1}(z, \bar{z})=\sqrt{-\partial \Phi(z, \bar{z})}, \quad \psi_{2}(z, \bar{z})=\sqrt{\bar{\partial} \Phi(z, \bar{z})},
$$

где $\Phi(z, \bar{z})=X^{2}(z, \bar{z})+i X^{1}(z, \bar{z})$, удовлетворяют уравнению (1) с $U(z, \bar{z})=$ $H(z, \bar{z}) D(z, \bar{z}) / 2$, где $H$ - среднял кривизна и $D^{2} d z d \bar{z}$ - метрика поверхности $X(W) \subset \mathbb{R}^{3}$.

Мы кратко изложим доказательство, следуя [16].

Условие конформности погружения выписывается в виде

$$
\left(\frac{\partial X^{1}}{\partial z}\right)^{2}+\left(\frac{\partial X^{2}}{\partial z}\right)^{2}+\left(\frac{\partial X^{3}}{\partial z}\right)^{2}=0
$$

Рассмотрим конформное погружение, удовлетворяющее условию (6), и по формулам (7) построим в окрестности точки $z_{0} \in W$ вектор-функцию $\psi$. Из (6) и (8) следует, что около $z_{0}$ подкоренные выражения из (7) не обращаются в нуль.

Выберем следующие базисы в касательных плоскостях к $X(W): e_{1}=$ $D^{-1} \partial X / \partial x, e_{2}=D^{-1} \partial X / \partial y$, где $D^{2} d z d \bar{z}$ — индуцированная метрика. Пусть $e_{3}=e_{1} \times e_{2}$. Деривационные формулы имеют вид

$$
\begin{aligned}
\frac{\partial^{2} X}{\partial x^{2}} & =\frac{\partial D}{\partial x} e_{1}-\frac{\partial D}{\partial y} e_{2}+D^{2} h_{11} e_{3}, \\
\frac{\partial^{2} X}{\partial x \partial y} & =\frac{\partial D}{\partial y} e_{1}+\frac{\partial D}{\partial x} e_{2}+D^{2} h_{12} e_{3}, \\
\frac{\partial^{2} X}{\partial y^{2}} & =-\frac{\partial D}{\partial x} e_{1}+\frac{\partial D}{\partial y} e_{2}+D^{2} h_{22} e_{3},
\end{aligned}
$$

где $h_{i j}$ - вторая фундаментальная форма.

Теперь лемма 2 доказывается подстановкой формул (9) в выражение для $\bar{\partial} \psi_{1}$ и $\partial \psi_{2}$ и последующими прямыми вычислениями.

ОПределЕниЕ 1 . Представление поверхности $\Sigma$, погруженной в $\mathbb{R}^{3}$, формулами (3) называется представлением Вейерштрасса.

Функция $U(z, \bar{z})$ вида (4) называется потенииалом поверхности $\Sigma$ c отмеченным конформныл параметром $z$, или потенциалом представления Вейерштрасса поверхности $\Sigma$.

Лемма 2 непосредственно влечет за собой [16] следующий результат:

ЛЕмма 3. Каждая $C^{2}$-регулярная поверхность, погруженная в $\mathbb{R}^{3}$, в окрестности каждой своей точки допускает представление Вейерштрасса.

2.2. Глобальное представление Вейерштрасса. Для того чтобы определить представление Вейерштрасса глобально для всей поверхности $\Sigma$, не- 
обходимо корректно определить расслоение, порожденное $\psi$ над $\Sigma$, и оператор $\mathscr{D}$, действующий в этом расслоении. Эта задача была решена в [16], и мы напомним ее решение.

Каждый тор конформно эквивалентен плоскому тору $\mathbb{C} / \Lambda$, где $\Lambda$ - peшетка ранга 2 .

ОПРЕДЕЛЕНИЕ 2. Тор $\Sigma$, погруженный в $\mathbb{R}^{3}$, имеет глобальное представление Вейерштрасса, если существуют вещественный потенциал $U$ и функции $\psi_{1}$ и $\psi_{2}$, определенные на универсальном накрытии тора $\Sigma$, т. е. на $\mathbb{C}$, такие, что

1) для $z \in \mathbb{C}$ и $\gamma \in \Lambda$

$$
U(z+\gamma)=U(z), \quad \psi_{j}(z+\gamma)=\varepsilon(\gamma) \psi_{j}(z), \quad \varepsilon(\gamma)= \pm 1
$$

2 ) вектор-функция $\psi$ удовлетворяет уравнению (1) и для подходящим образом выбранных координат в $\mathbb{R}^{3}$ определяет по формулам (3) погружение тора $\Sigma$.

Сфера $\Sigma$ с $g>1$ ручками конформно эквивалентна факторпространству $\mathscr{H} / \Lambda$, где $\mathscr{H}$ - верхняя полуплоскость Лобачевского и $\Lambda$ - решетка в $\operatorname{PSL}(2, \mathbb{R})$, группе изометрий пространства $\mathscr{H}$.

ОПРЕдЕлЕниЕ 3 . Сфера $\Sigma$ с $g(>1)$ ручками, погруженная в $\mathbb{R}^{3}$, имеет глобальное представление Вейерштрасса, если существуют вещественный потенциал $U$ и функции $\psi_{1}$ и $\psi_{2}$, определенные на универсальном накрытии сферы $\Sigma$, т.е. на $\mathscr{H}$, такие, что

1) для $z \in \mathscr{H}$ и $\gamma \in \Lambda$, представленных матрицей

$$
\left(\begin{array}{ll}
a & b \\
c & d
\end{array}\right), \quad a, b, c, d \in \mathbb{R}, a d-b c=1,
$$

имеют место равенства

$$
\begin{gathered}
U(\gamma(z))=|c z+d|^{2} U(z), \\
\psi_{1}(\gamma(z))=(c z+d) \psi_{1}(z), \quad \psi_{2}(\gamma(z))=(c \bar{z}+d) \psi_{2}(z) ;
\end{gathered}
$$

2) вектор-функция $\psi$ удовлетворяет (1) и для подходящим образом выбранных координат в $\mathbb{R}^{3}$ определяет по формулам (3) погружение сферы $\Sigma$.

Эти определения основываются на следующем утверждении.

ЛЕмма 4. Если локальное представление Вейерштрасса замкнутой ориентированной поверхности $\Sigma$ рода $g \geqslant 1$ гладко продолжается на все универсальное накрытие $\widetilde{\Sigma}$, то $U$ и ч удовлетворяют условиям (10) для $g=1$ $u$ (11) для $g \geqslant 2$.

Эта лемма следует из формул преобразования средней кривизны и метрики при замене координат. Теперь мы приходим к следующему результату [16].

ТЕОРЕмА 1. Если замкнутая ориентированная поверхность $\Sigma$ рода $\geqslant 1$ имеет глобальное представление Вейерштрасса, то $\psi$ является глобальным сечением спинорного расслоения над поверхностью постоянной кривизнь $\Sigma_{0}$, конформно эквивалентной $\Sigma$, и оператор Дирака (2) действует в этом расслоении. 
Следующая теорема демонстрирует важность этого представления.

ТЕоремА 2. Каждая $C^{3}$-регулярная замкнутая ориентированная поверхность $\Sigma$ рода $g \geqslant 1$, погруженная в $\mathbb{R}^{3}$, имеет глобальное представление Вейерuтрасса ${ }^{1}$.

ДокАЗАтЕльство. Согласно лемме 3, функции (7) определяют локальное представление Вейерштрасса около каждой точки, в которой $\partial \Phi \neq 0$. Для доказательства существования глобального представления достаточно корректно гладко продолжить эти функции на окрестности точек, в которых $\partial \Phi=0$. Согласно (8), последнее условие эквивалентно равенству $X_{z}^{3}=0$.

Выберем для $\Sigma$ такие координаты в $\mathbb{R}^{3}$, что все критические точки «функции высоты», расстояния до плоскости $X^{3}=0$, невырожденны по Морсу [13]. Вне критических точек определим $\psi$ по формулам (7) и покажем, что эти функции не имеют ветвлений в критических точках.

Пусть $q$ - критическая точка функции $X^{3}$ и $z$ - конформный параметр около $q$, такой, что $z(q)=0$. Так как точка невырожденна, то

$$
X^{3}(z, \bar{z})=\alpha z^{2}+\bar{\alpha} \bar{z}^{2}+\beta z \bar{z}+O\left(|z|^{3}\right)
$$

и $|\alpha|+|\beta| \neq 0$. Покажем, что (7) корректно определяет $\psi_{1}$ около $q$, и заметим, что эти рассуждения проходят и для $\psi_{2}$.

Taк как $\partial \Phi(q)=0$, то

$$
\frac{\partial X^{1}(q)}{\partial z}=i \frac{\partial X^{2}(q)}{\partial z} .
$$

Дифференцируя левую часть формулы (8) по z, мы выводим из (12) и (13), что

$$
(\partial \Phi)_{z}(q)=\frac{\partial^{2} X^{2}(q)}{\partial z^{2}}+i \frac{\partial^{2} X^{1}(q)}{\partial z^{2}}=0 .
$$

Аналогично, дифференцируя ее по $\bar{z}$, получаем

$$
(\partial \Phi)_{\bar{z}}(q)=\frac{\partial^{2} X^{2}(q)}{\partial z \partial \bar{z}}+i \frac{\partial^{2} X^{1}(q)}{\partial z \partial \bar{z}}=0 .
$$

Предположим, что $\alpha \neq 0$. Дважды дифференцируя левую часть формулы (8) по $z$, мы с учетом $(12),(13)$ и (14) получаем

$$
\left(\frac{\partial^{2} X^{3}(q)}{\partial z^{2}}\right)^{2}+\frac{\partial X^{2}(q)}{\partial z}\left(\frac{\partial^{3} X^{2}(q)}{\partial z^{3}}+i \frac{\partial^{3} X^{1}(q)}{\partial z^{3}}\right)=0
$$

и, так как поверхность регулярна, $X_{z}^{2}(q) \neq 0$. Следовательно,

$$
(\partial \Phi)_{z z}(q) \neq 0 \text {. }
$$

Если $\alpha=0$, то $\beta \neq 0$ и, дважды дифференцируя левую часть формулы (8) по $\bar{z}$, мы получаем

$$
(\partial \Phi)_{\bar{z} \bar{z}}(q) \neq 0
$$

1Эта теорема также верна для сфер, но этот случай мы рассмотрим отдельно. Для аналитических поверхностей существование глобального представления выводится из существования локального представления с помощью аналитического продолжения, и это было сделано в [16]. 
Из (15) и (16) мы заключаем, что $\partial \Phi$ имеет двойной нуль в $q$ и, если, например, (15) выполняется, $\partial \Phi=z^{2} \cdot f(z, \bar{z})$, где ветви $\sqrt{f}$ не имеют ветвления в $q$. Следовательно, $\sqrt{-\partial \Phi}$ не имеет ветвления в $q$.

Так как мы рассматривали произвольную критическую точку функции $X^{3}$, то каждая ветвь $\sqrt{-\partial \Phi}$ корректно определена и не имеет ветвления. Следовательно, на универсальном накрытии поверхности $\Sigma$ вектор-функция $\psi$ корректно определена формулой (7).

Теорема 2 доказана.

\section{§3. Спектр представления Вейерштрасса}

Пусть $\Sigma$ - тор, погруженный в $\mathbb{R}^{3}$ и конформно эквивалентный плоскому тору $\mathbb{C} / \Lambda$. Предположим, что он имеет глобальное представление Вейерштрасса, что по теореме 2 выполняется, если он $C^{3}$-регулярен:

1) существует функция $U(z): \mathbb{C} \rightarrow \mathbb{R}$, такая, что $U(z+\gamma)=U(z)$ для $z \in \mathbb{C}, \gamma \in \Lambda$

2) существуют постоянные $\varepsilon_{1}$ и $\varepsilon_{2}$, такие, что $\varepsilon_{j} \in\{0,1\}$, и они определяют спинорное расслоение над $\mathbb{C} / \Lambda$;

3) существует функция $\psi$, такая, что $\mathscr{D} \psi=0$, и для нее выполняются условия периодичности (10).

Согласно лемме 1 , каждое решение уравнения (1) определяет погружение поверхности в $\mathbb{R}^{3}$. Мы рассмотрим «линейный базис» для семейства таких поверхностей, а именно множество функций Флоке.

ОПРЕДЕлЕНИЕ 4. Функция $\psi: \mathbb{C} \rightarrow \mathbb{C}$ называется функцией Флоке ( $с$ нулевым собственным значением) оператора $\mathscr{D}($ см. (2)) с квазиимпульсами $\left(k_{1}, k_{2}\right)$, если $\mathscr{D} \psi=0$ и

$$
\psi(z+\gamma)=\exp \left(2 \pi i\left(\operatorname{Re} \gamma \cdot k_{1}+\operatorname{Im} \gamma \cdot k_{2}\right)\right) \psi(z)
$$

для $\gamma \in \Lambda$.

Заметим, что функция $\psi$, удовлетворяющая (17), имеет вид

$$
\psi(z)=\exp \left(2 \pi i\left(x k_{1}+y k_{2}\right)\right) \varphi(z)
$$

где $\varphi(z)$ периодична относительно $\Lambda$. Следующая лемма очевидна.

ЛЕмма 5. Функиия $\psi$ вида (18) удовлетворяет уравнению $\mathscr{D} \psi=0$ тогда и только тогда, когда

$$
\mathscr{D}_{k} \varphi=0
$$

әде

$$
\mathscr{D}_{k}=\left(\begin{array}{cc}
0 & \partial \\
-\bar{\partial} & 0
\end{array}\right)+\left(\begin{array}{cc}
U & \pi\left(k_{2}+i k_{1}\right) \\
\pi\left(k_{2}-i k_{1}\right) & U
\end{array}\right) .
$$

Выберем постоянную $C$ так, чтобы оператор

$$
\mathscr{A}=\left[\left(\begin{array}{cc}
0 & \partial \\
-\bar{\partial} & 0
\end{array}\right)+\left(\begin{array}{cc}
C & 0 \\
0 & C
\end{array}\right)\right]
$$


был обратим на $L_{2}(\mathbb{C} / \Lambda)$. В этом случае уравнение $(19)$ имеет решения из $L_{2}(\mathbb{C} / \Lambda)$ тогда и только тогда, когда уравнение

$$
\left[1+\left(\begin{array}{cc}
U-C & \pi\left(k_{2}+i k_{1}\right) \\
\pi\left(k_{2}-i k_{1}\right) & U-C
\end{array}\right) \mathscr{A}^{-1}\right] \xi=0
$$

разрешимо в $L_{2}(\mathbb{C} / \Lambda)$. Так как оператор $\mathscr{A}^{-1}$ повышает гладкость, то по теореме вложения Соболева он компактен. Поскольку $U$ непрерывна, оператор умножения на

$$
\left(\begin{array}{cc}
U-C & \pi\left(k_{2}+i k_{1}\right) \\
\pi\left(k_{2}-i k_{1}\right) & U-C
\end{array}\right)
$$

ограничен. Следовательно, верна

Лемма 6. Пучок операторов

$$
\mathscr{D}_{k} \circ \mathscr{A}^{-1}-1=\left(\begin{array}{cc}
U-C & \pi\left(k_{2}+i k_{1}\right) \\
\pi\left(k_{2}-i k_{1}\right) & U-C
\end{array}\right) \mathscr{A}^{-1}
$$

полиномиален по $k_{1}$ u $k_{2}$ и состоит из компактных операторов из $L_{2}(\mathbb{C} / \Lambda)$ в $L_{2}(\mathbb{C} / \Lambda)$.

Теперь с использованием полиномиальной альтернативы Фредгольма [8] мы получаем такой результат:

Теорема 3. Существует аналитическое подмножество $\Gamma_{U} \subset \mathbb{C}^{2}$ положительной коразмерности, такое, что уравнение $(20)$ разрешимо в $L_{2}(\mathbb{C} / \Lambda)$ тогда и только тогда, когда $\left(k_{1}, k_{2}\right) \in \Gamma_{U}$.

Это подмножество называется (нулевым) спектром Флоке оператора $\mathscr{D}$.

ДокаЗАтЕльство теоремы 3. По теореме Келдыша [8], если $A_{\mu}$ - полиномиальный по $\mu \in \mathbb{C}^{n}$ пучок компактных операторов, то множество таких $\mu$, что уравнение $\left(1+A_{\mu}\right) \xi=0$ разрешимо, образует аналитическое подмногообразие в $\mathbb{C}^{n}$.

Докажем, что это подмножество имеет положительную коразмерность. Пусть $k_{1}=0$. При $\left|k_{2}\right| \rightarrow \infty$ влияние ограниченного потенциала становится пренебрежительно малым и в пределе (19) вырождается в

$$
\left(\left(\begin{array}{cc}
0 & \partial \\
-\bar{\partial} & 0
\end{array}\right)+\left(\begin{array}{cc}
0 & \pi k_{2} \\
\pi k_{2} & 0
\end{array}\right)\right) \xi=0 .
$$

Методами теории возмущений из последнего уравнения выводится, что существует $k_{2}$ (с достаточно большим $\left|k_{2}\right|$ ), для которого (19) неразрешимо в $L_{2}(\mathbb{C} / \Lambda)$. Теорема 3 доказана.

Более глубокие аналитические свойства спектра Флоке (представление его в виде множества нулей целой функции) могут быть получены методами [12].

Из (18) следует, что $\Gamma_{U}$ инвариантен относительно действия двойственной решетки $\Lambda^{*}$ :

$$
k_{1} \rightarrow k_{1}+\operatorname{Re} \gamma^{*}, \quad k_{2} \rightarrow k_{2}+\operatorname{Im} \gamma^{*}, \quad \gamma^{*} \in \Lambda^{*} .
$$

Напомним, что $\Lambda^{*}$ состоит из $\gamma^{*} \in \mathbb{C}$, таких, что $\left(\gamma, \gamma^{*}\right)=\operatorname{Re} \gamma \cdot \operatorname{Re} \gamma^{*}+$ $\operatorname{Im} \gamma \cdot \operatorname{Im} \gamma^{*} \in \mathbb{Z}$ для всех $\gamma \in \Lambda$. Также ясно, что определение $\Gamma_{U}$ не зависит от выбора конформного параметра $z$ на торе. 
ОПРЕДЕЛЕНИЕ 5 . Комплексная поверхность $W_{\Sigma}$, определенная как факторпространство спектра Флоке $\Gamma_{U}$ по действию (22), называется спектром Вейерштрасса тора $\Sigma$. Род нормализации поверхности $W_{\Sigma}$ называется сnектральнылм родом тора $\Sigma$.

Этот спектр был введен автором, указавшим на его связь с функционалом Уиллмора, интегралом от квадрата средней кривизны, как простейшим интегралом Крускала. Для торов вращения это было детально проанализировано в [17].

Более эффективное определение спектра Флоке для многомерных операторов дается теорией возмущений. Для оператора Шрёдингера и для $\partial_{y}-\partial_{x}^{2}$ это было сделано Кричевером (см. [11], где подход к определению спектральных кривых с помощью теоремы Келдыша был тоже отмечен со ссылкой на нашу неопубликованную работу).

Функционал Уиллмора $\mathscr{W}$ конформно инвариантен в следующем смысле. Пусть $g: \mathbb{R}^{3} \rightarrow \mathbb{R}^{3}$ - конформное преобразование, сохраняющее замкнутую поверхность $\Sigma$ в компактной области. Тогда $\mathscr{W}(\Sigma)=\mathscr{W}(g(\Sigma))$. Автор предположил, что весь спектр Вейерштрасса конформно инвариантен в этом смысле. Довольно скоро после ее постановки два различных доказательства этой гипотезы были получены Пинкаллем, использовавшим методы [7], и Гриневичем и Шмидтом [5].

Связь спектра Вейерштрасса со спектральными кривыми специальных солитонных торов $[6,14]$ будет обсуждена отдельно.

\section{§4. Конечнозонные плоскости и торы}

В двух следующих параграфах мы иногда даем только наброски доказательств, которые привычны для конечнозонной теории. Кроме того, для одномерного предела (2) вывод тэта-функциональных формул изложен в [15], а симметрии Г обсуждаются в [2].

Рассмотрим более общий оператор

$$
L=\left(\begin{array}{cc}
0 & \partial \\
-\bar{\partial} & 0
\end{array}\right)+\left(\begin{array}{cc}
U & 0 \\
0 & V
\end{array}\right)
$$

где $U$ и $V$ периодичны по отношению к решетке ранга два $\Lambda \subset \mathbb{C}$. Для этого оператора выполняется аналог теоремы 3 , и $L$ называется конечнозонным (на нулевом уровне энергии), если нормализация его нулевого спектра Флоке является компактной римановой поверхностью с двумя удаленными точками. Но мы называем $L$ конечнозонным, если он имеет вид, описанный в следующей теореме ${ }^{2}$ :

ПрЕДЛОЖЕНИЕ 1. Пусть Г- - омпактная риманова поверхность рода $g$, $\infty_{ \pm}$- пара различных точек на ней, $k_{ \pm}^{-1}$ - локальные параметрь в их окрестностлх, такие, ито $k_{ \pm}^{-1}\left(\infty_{ \pm}\right)=0$, и $D$ - неспециальньй эффективный дивизор степени $g+1$ на $\Gamma \backslash\left\{\infty_{ \pm}\right\}$, m.е. $D=P_{1}+\cdots+P_{g+1}$, где $P_{i} \in \Gamma \backslash\left\{\infty_{ \pm}\right\}$. Тогда

${ }^{2}$ Ясно, что эквивалентность этих определений для гладких потенциалов может быть обоснована с помощью теории возмущений [11]. 
1. Существует единственная вектор-функиия $\psi(z, \bar{z}, P)=\left(\psi_{1}, \psi_{2}\right)$, где $z \in \mathbb{C}$, такая, ито $\psi$ мероморфна по $P$ на $\Gamma \backslash\left\{\infty_{ \pm}\right\}$и имеет полюсы только в $D$,

$$
\begin{aligned}
& \psi=\exp \left(k_{+} z\right)\left[\left(\begin{array}{l}
1 \\
0
\end{array}\right)+\left(\begin{array}{l}
\xi_{11}^{+} / k_{+} \\
\xi_{21}^{+} / k_{+}
\end{array}\right)+O\left(k_{+}^{-2}\right)\right] \quad \text { npu } P \rightarrow \infty_{+}, \\
& \psi=\exp \left(k_{-} \bar{z}\right)\left[\left(\begin{array}{l}
0 \\
1
\end{array}\right)+\left(\begin{array}{l}
\xi_{11}^{-} / k_{-} \\
\xi_{21}^{-} / k_{-}
\end{array}\right)+O\left(k_{-}^{-2}\right)\right] \quad \text { nрu } P \rightarrow \infty_{-} .
\end{aligned}
$$

2. Существует единственньй оператор $L$ вида (23), такой, что $L \psi=0$. Его потенииальи имеют вид

$$
U=-\xi_{21}^{+}, \quad V=\xi_{11}^{-} .
$$

Это предложение - частный случай общей теоремы о единственности функции Бейкера-Ахиезера [10].

В дальнейшем, чтобы избежать громоздкости, мы ограничимся случаем, когда $Г$ не имеет сингулярных точек. Хотя в геометрии поверхностей важен случай, когда Г имеет двойные точки (например, тор Клиффорда [17]), он получается в пределе из рассмотренных ниже.

Выберем базис $\alpha_{1}, \ldots, \alpha_{g}, \beta_{1}, \ldots, \beta_{g}$ в $H_{1}(\Gamma)$ с формой пересечения $\alpha_{j} \circ \beta_{k}=\delta_{j k}, \alpha_{j} \circ \alpha_{k}=\beta_{j} \circ \beta_{k}=0$. Этому базису соответствует единственный базис голоморфных дифференциалов $\omega_{1}, \ldots, \omega_{g}$, нормированных условием $\int_{\alpha_{k}} \omega_{j}=\delta_{j k}$. Определим матрицу периодов $\Omega$ и тэта-функцию поверхности $\Gamma$ :

$$
\Omega_{j k}=\int_{\beta_{k}} \omega_{j}, \quad \vartheta(u)=\sum_{N \in \mathbb{Z}^{g}} \exp \{\pi i((\Omega N, N)+2(N, u))\},
$$

где $u \in \mathbb{C}^{g}$. Зафиксировав точку $P_{0} \in \Gamma \backslash\left\{\infty_{ \pm}\right\}$, определим отображение Абеля $A(P)=\left(\int_{P_{0}}^{P} \alpha_{1}, \ldots, \int_{P_{0}}^{P} \alpha_{g}\right)$ из поверхности $\Gamma$ в ее многообразие Якоби $J(\Gamma)=\mathbb{C}^{g} /\left\{M+\Omega N: M, N \in \mathbb{Z}^{g}\right\}$.

Обозначим через $\eta_{l}^{ \pm}$единственный мероморфный дифференциал, имеющий единственный полюс в $\infty_{ \pm}$с лорановской частью $d k_{ \pm}^{l}$ и нормированный условием $\int_{\alpha_{j}} \eta_{l}^{ \pm}=0$ при $j=1, \ldots, g$. Каждому $\eta_{l}^{ \pm}$соответствует вектор $\beta$-периодов $\left(U_{l}^{ \pm}\right)^{j}=(2 \pi i)^{-1} \int_{\beta_{j}} \eta_{l}^{ \pm}$.

Существуют эффективные дивизоры $Q_{1}+\cdots+Q_{g}$ и $R_{1}+\cdots+R_{g}$, такие, что выполняются линейные эквивалентности

$$
D=P_{1}+\cdots+P_{g+1} \sim Q_{1}+\cdots+Q_{g}+\infty_{-} \sim R_{1}+\cdots+R_{g}+\infty_{+} .
$$

Положим $Q_{g+1}=\infty_{-}$и $R_{g+1}=\infty_{+}$и обозначим $A\left(Q_{1}\right)+\cdots+A\left(Q_{g}\right)$ и $A\left(R_{1}\right)+\cdots+A\left(R_{g}\right)$ через $A(Q)$ и $A(R)$ соответственно.

Обозначим через $\delta$ вектор римановых констант, определяемый следующим образом: для точки общего положения $u \in J(\Gamma)$ многозначная функция $\vartheta(A(P)-u)$ имеет нули в точности в $g$ точках $S_{1}, \ldots, S_{g}$, таких, что $u+\delta=A\left(S_{1}\right)+\cdots+A\left(S_{g}\right)$. Мы также выберем $\varepsilon$, нечетный полупериод функции $\vartheta$, т.е. $\vartheta(\varepsilon)=0$ и $2 \varepsilon \equiv 0$ на $J(\Gamma)$. 
ПРЕДЛОЖЕНИЕ 2. Функиия $\psi$ из предложения 1 имеет вид

$$
\begin{aligned}
& \psi_{1}(z, \bar{z}, P)=\exp \left(z\left(\int_{P_{0}}^{P} \eta_{1}^{+}-a_{1}^{+}\right)+\bar{z}\left(\int_{P_{0}}^{P} \eta_{1}^{-}-b_{1}^{-}\right)\right) \\
& \times \frac{\vartheta\left(A(P)+F_{1}(z, \bar{z})\right)}{\vartheta(A(P)+\delta-A(Q))} \cdot \frac{\vartheta\left(A\left(\infty_{+}\right)+\delta-A(Q)\right)}{\vartheta\left(A\left(\infty_{+}\right)+F_{1}(z, \bar{z})\right)} \\
& \times \frac{\prod^{g+1} \vartheta\left(\varepsilon+A(P)-A\left(Q_{j}\right)\right) \cdot \vartheta\left(\varepsilon+A\left(\infty_{+}\right)-A\left(P_{j}\right)\right)}{\prod^{g+1} \vartheta\left(\varepsilon+A(P)-A\left(P_{j}\right)\right) \cdot \vartheta\left(\varepsilon+A\left(\infty_{+}\right)-A\left(Q_{j}\right)\right)}, \\
& \psi_{2}(z, \bar{z}, P)=\exp \left(z\left(\int_{P_{0}}^{P} \eta_{1}^{+}-b_{1}^{+}\right)+\bar{z}\left(\int_{P_{0}}^{P} \eta_{1}^{-}-a_{1}^{-}\right)\right) \\
& \times \frac{\vartheta\left(A(P)+F_{2}(z, \bar{z})\right)}{\vartheta(A(P)+\delta-A(R))} \cdot \frac{\vartheta\left(A\left(\infty_{-}\right)+\delta-A(R)\right)}{\vartheta\left(A\left(\infty_{-}\right)+F_{2}(z, \bar{z})\right)} \\
& \times \frac{\prod^{g+1} \vartheta\left(\varepsilon+A(P)-A\left(R_{j}\right)\right) \cdot \vartheta\left(\varepsilon+A\left(\infty_{-}\right)-A\left(P_{j}\right)\right)}{\prod^{g+1} \vartheta\left(\varepsilon+A(P)-A\left(P_{j}\right)\right) \cdot \vartheta\left(\varepsilon+A\left(\infty_{-}\right)-A\left(R_{j}\right)\right)},
\end{aligned}
$$

где постоянные $a_{1}^{ \pm}$и $b_{1}^{ \pm}$определяются условиями

$$
\begin{array}{ll}
\int_{P_{0}}^{P} \eta_{1}^{ \pm}-a_{1}^{ \pm}=k_{ \pm}+O\left(k_{ \pm}^{-1}\right) & \text { около } \infty_{ \pm} \\
\int_{P_{0}}^{P} \eta_{1}^{ \pm}-b_{1}^{ \pm}=O\left(k_{\mp}^{-1}\right) & \text { около } \infty_{\mp}
\end{array}
$$

$u$

$$
F_{1}(z, \bar{z})=U_{1}^{+} z+U_{1}^{-} \bar{z}+\delta-A(Q), \quad F_{2}(z, \bar{z})=U_{1}^{+} z+U_{1}^{-} \bar{z}+\delta-A(R) .
$$

Потенциаль $U$ и $V$ имеют вид

$$
U=c_{1} \exp \left(z\left(a_{1}^{+}-b_{1}^{+}\right)+\bar{z}\left(b_{1}^{-}-a_{1}^{-}\right)\right) \frac{\vartheta\left(A\left(\infty_{+}\right)+F_{2}(z, \bar{z})\right)}{\vartheta\left(A\left(\infty_{-}\right)+F_{2}(z, \bar{z})\right)},
$$

гдe

$$
\begin{aligned}
c_{1}=- & \frac{\prod^{g+1} \vartheta\left(\varepsilon+A\left(\infty_{-}\right)-A\left(P_{j}\right)\right)}{\prod^{g+1} \vartheta\left(\varepsilon+A\left(\infty_{+}\right)-A\left(P_{j}\right)\right) \cdot \vartheta\left(\varepsilon+A\left(\infty_{-}\right)-A\left(R_{j}\right)\right)} \\
& \times \prod_{j=l}^{g} \vartheta\left(\varepsilon+A\left(\infty_{+}\right)-A\left(R_{l}\right)\right) \cdot \sum\left(U_{1}^{+}\right)^{m} \frac{\partial \vartheta(\varepsilon)}{\partial u^{m}},
\end{aligned}
$$

$u$

$$
V=c_{2} \exp \left(z\left(b_{1}^{+}-a_{1}^{+}\right)+\bar{z}\left(a_{1}^{-}-b_{1}^{-}\right)\right) \frac{\vartheta\left(A\left(\infty_{-}\right)+F_{1}(z, \bar{z})\right)}{\vartheta\left(A\left(\infty_{+}\right)+F_{1}(z, \bar{z})\right)},
$$

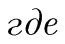

$$
\begin{gathered}
c_{2}=\frac{\prod^{g+1} \vartheta\left(\varepsilon+A\left(\infty_{+}\right)-A\left(P_{j}\right)\right)}{\prod^{g+1} \vartheta\left(\varepsilon+A\left(\infty_{-}\right)-A\left(P_{j}\right)\right) \cdot \vartheta\left(\varepsilon+A\left(\infty_{+}\right)-A\left(Q_{j}\right)\right)} \\
\times \prod_{j=l}^{g} \vartheta\left(\varepsilon+A\left(\infty_{-}\right)-A\left(Q_{l}\right)\right) \cdot \sum\left(U_{1}^{-}\right)^{m} \frac{\partial \vartheta(\varepsilon)}{\partial u^{m}} .
\end{gathered}
$$


Здесь мы предполагаем, что пути, соединяющие $P_{0}$ и малые окрестности бесконечностей, и пути, входящие в определение $A(P)$ и $A\left(\infty_{ \pm}\right)$- одни и те же и для некоторых их гомотопических классов выполняются разложения (27).

ДОКАЗАТЕЛЬСТВо ПРЕДлОЖЕНИЯ 2. Формулы для $\psi$ проверяются с помощью периодических свойств тэта-функций и теоремы Римана о нулях тэтафункций [4].

Выведем (28) и (29). Например, около $\infty_{-}$функция $\psi_{1}$ разлагается в произведение $\vartheta\left(\varepsilon+A(P)-A\left(\infty_{-}\right)\right) \cdot H(z, \bar{z}, P) \cdot \exp \left(k_{-} \bar{z}\right)$ и известно, что $\partial A(P) / \partial k_{-}^{-1}=U_{1}^{-}$в $\infty_{-}$. Следовательно $\xi_{11}^{-}=H\left(z, \bar{z}, \infty_{-}\right) \cdot \sum\left(U_{1}^{-}\right)^{j} \partial \vartheta(\varepsilon) / \partial u^{j}$.

Предложение доказано.

ПРЕДЛОЖЕНИЕ 3. Пусть спектральные даннье $\left(\Gamma, \infty_{ \pm}, k_{ \pm}, D\right)$ конечнозонного оператора $L$ вида (23) (см. предложение 1) удовлетворяют следуюшим условиям:

(i) существует голоморфная инволюиия $\sigma: \Gamma \rightarrow \Gamma$, такал, ито $\sigma\left(\infty_{ \pm}\right)=$ $\infty_{ \pm}, \sigma\left(k_{ \pm}\right)=-k_{ \pm}$и существует мероморфньй дифференциал $\omega$ на нулями в $D+\sigma(D)$ и двумл полюсами в $\infty_{ \pm}$с главныли частями $\left( \pm k_{ \pm}^{2}+\right.$ $\left.O\left(k_{ \pm}^{-1}\right)\right) d k_{ \pm}^{-1}$

(ii) существует антиголоморфная инволючия $\tau: \Gamma \rightarrow \Gamma$, такая, ито $\tau\left(\infty_{ \pm}\right)=\infty_{\mp}, \tau\left(k_{ \pm}\right)=-\bar{k}_{\mp}$ и сушествует мероморфный дифференциал $\widetilde{\omega}$ на Г с нулями в $D+\tau(D)$ и двумл полюсами в $\infty_{ \pm}$с главными частями $\left(k_{ \pm}^{2}+O\left(k_{ \pm}^{-1}\right)\right) d k_{ \pm}^{-1}$.

Тогда $L$ имеет вид (2) с вещественным потенииалом $U$, m.е. $U=V=\bar{U}$.

ДокаЗАтЕльство. Возьмем мероморфный дифференциал $\psi_{1}(P) \psi_{2}(\sigma(P)) \omega$ с полюсами только в $\infty_{ \pm}$. Сумма его вычетов равна $\xi_{21}^{+}+\xi_{11}^{-}$. Так как она равна нулю, то, согласно (26), это влечет за собой равенство $U=V$.

Теперь рассмотрим дифференциалы $\psi_{1}(P) \overline{\psi_{1}(\tau(P))} \widetilde{\omega}$ и $\psi_{2}(P) \overline{\psi_{2}(\tau(P))} \widetilde{\omega}$ и, вычисляя, как выше, суммы их вычетов, заключаем, что $U=\bar{U}$ и $V=\bar{V}$. Предложение доказано.

ПрЕДлОЖЕНИЕ 4. 1) Пусть спектральные даннье $\left(\Gamma, \infty_{ \pm}, k_{ \pm}, D\right)$ удовлетворяют условиям предложения $3 ;$ тогда для любьх $n$ точек $Q_{1}, \ldots, Q_{n} \in$ $\Gamma \backslash\left\{\infty_{ \pm}, P_{1}, \ldots, P_{g}\right\}$ и любых $n$ постоянньх $a_{1}, \ldots, a_{n} \in \mathbb{C}$ вектор-функиия $\psi(z, \bar{z})=a_{1} \psi\left(z, \bar{z}, Q_{1}\right)+\cdots+a_{n} \psi\left(z, \bar{z}, Q_{n}\right)$ определяет по формулам (3) поверхность, погруженную в $\mathbb{R}^{3}$.

2) Если $\psi(z+\gamma)= \pm \psi(z)$ для $z \in \mathbb{C} u \gamma \in \Lambda$, где $\Lambda \subset \mathbb{C}-$ решетка ранга два в $\mathbb{C}$, то это погружение сворачивается в погружение тора $\mathbb{C} / \Lambda$ тогда и только тогда, когда

$$
\int_{\mathbb{C} / \Lambda} \psi_{1}^{2} d z \wedge d \bar{z}=\int_{\mathbb{C} / \Lambda} \psi_{2}^{2} d z \wedge d \bar{z}=\int_{\mathbb{C} / \Lambda} \psi_{1} \bar{\psi}_{2} d z \wedge d \bar{z}=0 .
$$

ДоказАТЕЛьСтво. Первое утверждение очевидно. Второе утверждение достаточно ясно для торов вращения [17] и было распространено на общий случай М. Шмидтом. 
Пусть $\left(\gamma_{1}, \gamma_{2}\right)$ - положительно ориентированный базис решетки $\Lambda$. Согласно $(3), X^{1}+i X^{2}=i \int\left(\bar{\psi}_{2}^{2} d z-\bar{\psi}_{1}^{2} d \bar{z}\right)$. Мы также имеем

$$
\begin{aligned}
& d\left(x\left(\bar{\psi}_{2}^{2} d z-\bar{\psi}_{1}^{2} d \bar{z}\right)\right)=i\left(\bar{\psi}_{1}^{2}+\bar{\psi}_{2}^{2}\right) d x \wedge d y \\
& d\left(y\left(\bar{\psi}_{2}^{2} d z-\bar{\psi}_{1}^{2} d \bar{z}\right)\right)=\left(\bar{\psi}_{1}^{2}-\bar{\psi}_{2}^{2}\right) d x \wedge d y .
\end{aligned}
$$

Из теоремы Стокса следует, что

$$
\begin{aligned}
& \int_{\mathbb{C} / \Lambda}\left(\bar{\psi}_{1}^{2}+\bar{\psi}_{2}^{2}\right) d z^{\prime} \wedge d \bar{z}^{\prime}=-2\left(\operatorname{Re} \gamma_{1} \int_{z}^{z+\gamma_{2}} \tau-\operatorname{Re} \gamma_{2} \int_{z}^{z+\gamma_{1}} \tau\right), \\
& \int_{\mathbb{C} / \Lambda}\left(\bar{\psi}_{1}^{2}-\bar{\psi}_{2}^{2}\right) d z^{\prime} \wedge d \bar{z}^{\prime}=2 i\left(\operatorname{Im} \gamma_{1} \int_{z}^{z+\gamma_{2}} \tau-\operatorname{Im} \gamma_{2} \int_{z}^{z+\gamma_{1}} \tau\right),
\end{aligned}
$$

где $\tau=\bar{\psi}_{2}^{2} d z^{\prime}-\bar{\psi}_{1}^{2} d \bar{z}^{\prime}$. Следовательно, $X^{1}+i X^{2}$ периодична относительно $\Lambda$, если и только если первые два равенства из (30) выполняются. Эквивалентность последнего $\Lambda$-периодичности $X^{3}$ доказывается аналогично. Предложение доказано ${ }^{3}$.

Конечнозонные потенциалы могут быть выписаны в терминах тэта-функций Прима накрытия $\Gamma \rightarrow \Gamma / \sigma$, как и в случае двумерного оператора Шрёдингера $[19,20]$, но, в отличие от этого случая, мы не можем утверждать, что инволюция $\sigma$ имеет только две гладкие неподвижные точки.

\section{§5. Функционал Уиллмора}

Следуюшая гипотеза Уиллмора широко известна:

для торов, погруженных в $\mathbb{R}^{3}$, минимум Функционала Уиллмора

$$
\mathscr{W}(\Sigma)=\int_{\Sigma} H^{2} d \mu
$$

где $d \mu-$-индуцированнал мера Лиувиля, равен $2 \pi^{2}$ и достигаетсл на торе Клиффорда и его образах под действием конформных преобразований пространства $\mathbb{R}^{3}$.

Тор Клиффорда получается вращением окружности радиуса 1 вокруг оси, лежащей в той же плоскости, что и окружность, и отстоящей от окружности на расстояние $\sqrt{2}$.

K настоящему времени эта гипотеза доказана только в некоторых частных случаях (см. обзор их в [16]).

Согласно (4), для тора $\Sigma \subset \mathbb{R}^{3}$ простейший интеграл Крускала

$$
4 \int_{\mathbb{C} / \Lambda} U^{2}(z, \bar{z}) d x d y
$$

\footnotetext{
${ }^{3}$ Построенные поверхности могут иметь сингулярности, которые находятся в точности в тех точках, где $\left|\psi_{1}\right|^{2}+\left|\psi_{2}\right|^{2}=0$. Для $C^{2}$-регулярных поверхностей условия периодичности (30) достаточно наглядны, так как, согласно (7) и $(8), \psi_{1}^{2}=-\partial\left(X^{2}+i X^{1}\right)$, $\psi_{2}^{2}=\bar{\partial}\left(X^{2}+i X^{1}\right)$ и $\psi_{1} \bar{\psi}_{2}=\partial X^{3}$ всюду.
} 
где $U: \mathbb{C} / \Lambda \rightarrow \mathbb{C}$ - потенциал его представления Вейерштрасса, совпадает с $\mathscr{W}(\Sigma)[16]$. Это ведет к гипотезе, что

для фиксированньх конформных классов торов минимумы функционала $\mathscr{W}$ достигаются на торах наименьшего спектрального радиуса.

Это соответствует гипотезе Уиллмора, так как спектральный род тора Клиффорда равен нулю (это выводится из его представления Вейерштрасса, найденного в [17]).

Представление Вейерштрасса дает физическое объяснение для нижних границ функционала $\mathscr{W}$ : дело в том, что для малых возмущений нулевого потенциала $U=0$ плоскости вида (3) не сворачиваются в торы и, так как для $U$ функционал Уиллмора является квадратом его $L_{2}$-нормы, нижняя оценка показывает, насколько большим должно быть возмущение, чтобы плоскости свернулись в торы.

ЗАКЛЮЧИТЕЛЬНОЕ ЗАМЕЧАНИЕ. Часть этой работы была проделана во время пребывания автора в IHES (январь 1997 г.). Автор благодарит П. Гриневича, У. Пинкалля и М. Шмидта за полезные обсуждения.

\section{ЛитературА}

1. Bobenko A. Surfaces in terms of 2 by 2 matrices. Old and new integrable cases. In: Harmonic maps and integrable systems, Aspects Math. E23, Vieweg, 1994, pp. 83127.

2. Дубровин Б. А. Матричные конечнозонные операторы. Итоги науки и техники, Соврем. проблемы математики. Новейшие достижения, т. 23, 1983, с. 33-78.

3. Eisenhart L. P. A treatise on the differential geometry of curves and surfaces. Dover, New York, 1909.

4. Fay J. Theta functions on Riemann surfaces. Lect. Notes in Math., Vol. 352, Springer-Verlag, 1973.

5. Grinevich P., Schmidt M. U. Conformal invariant functionals on tori in $\mathbb{R}^{3}$. Гoтовится к печати в J. Geom. Phys.

6. Hitchin $N$. Harmonic maps from a 2-torus into the 3-sphere. J. Differential Geom., 31, 627-710 (1990).

7. Kamberov G., Pedit F., Pinkall U. Bonnet pairs and isothermic surfaces. Готовится к печати в Duke Math. J.

8. Келдыш M. B. О собственных значениях и собственных функциях некоторых классов несамосопряженных уравнений. ДАН, 77, 11-14 (1951).

9. Konopelchenko B. G. Induced surfaces and their integrable dynamics. Stud. Appl. Math., 96, 9-52 (1996).

10. Кричевер И. М. Методы алгебраической геометрии в теории нелинейных уравнений. УМН, 32, вып. 6, 183-206 (1977).

11. Кричевер И. М. Спектральная теория двумерных периодических операторов и ее приложения. УМН, 44, вып. 2, 121-184 (1989).

12. Кучмент П. А. Теория Флоке для уравнений в частных производных. УМН, 37, вып. 4, 3-52 (1982).

13. Milnor J. Morse Theory. Ann. of Math. Studies, Princeton Univ. Press, Princeton, 1963.

14. Pinkall U., Sterling I. On the classification of constant mean curvature tori. Ann. of Math., 130, 407-451 (1989).

15. Previato E. Hyperelliptic quasi-periodic and soliton solutions of the nonlinear Schrödinger equation. Duke Math. J., 52, 329-377 (1985). 
16. Taimanov I. A. Modified Novikov-Veselov equation and differential geometry of surfaces. In: Amer. Math. Soc. Transl. Ser. 2, Vol. 179, 1997, pp. 133-151.

17. Taimanov I. A. Surfaces of revolution in terms of solitons. Ann. Global Anal. Geom., 15, 419-435 (1997).

18. Тайманов И. А. Глобальное представление Вейерштрасса и его спектр. УМН, 52, вып. 6, 187-188 (1997).

19. Веселов А. П., Новиков С. П. Конечнозонные двумерные операторы Шрёдингера. Потенциальные операторы. ДАН, 279, 784-788 (1984).

20. Веселов А. П., Новиков С. П. Конечнозонные двумерные потенциальные операторы Шрёдингера. Точные формулы и эволюционные уравнения. ДАН, $\mathbf{2 7 9}$, 20-24 (1984).

Институт математики СО РАН, Новосибирск e-mail: taimanov@math.nsc.ru

Поступило в редакцию 30 декабря 1997 г. 